\title{
The transcription factor STAT5 binds to distinct super-enhancer sites and controls Lrrc32 expression in a prominent autoimmune and allergic disease risk locus
}

\author{
Lothar Hennighausen and Hye Kyung Lee
}

Laboratory of Genetics and Physiology, National Institute of Diabetes and Digestive and Kidney Diseases, US National Institutes of Health, Bethesda, Maryland 20892, USA.

${ }^{*}$ Correspondence to: L.H (lotharh@niddk.nih.gov) and H.K.L (hyekyung.lee@nih.gov)

\section{Summary}

Genetic variants associated with diseases are enriched in genomic sequences linked to regulatory regions, such as enhancers, super-enhancers and possibly repressors, that control nearby and distant genes. A known allergic and autoimmune risk locus at chromosome $11 \mathrm{q} 13.5^{1,2}$ is associated with the $L R R C 32$ gene, which encodes GARP, a protein critical for TGF- $\beta$ delivery ${ }^{3}$. This region coincides with a candidate enhancer that was predicted by the presence of activating chromatin marks and contains a polymorphism significantly associated with GARP expression on $\mathrm{CD} 4^{+} \mathrm{CD} 127^{-} \mathrm{CD} 25^{+}$ $\mathrm{T}_{\text {reg }}$ cells ${ }^{4}$. In the mouse, binding of the cytokine-induced transcription factor STAT5 was detected at two sites within the expansive candidate enhancer region and a 2.3 $\mathrm{kb}$ deletion resulted in reduced $L$ rrc32 expression ${ }^{4}$. However, a clear definition of the enhancer units controlled by STAT5 and a functional understanding of STAT5 in the regulation of Lrrc32 are needed. Here we use high-resolution ChIP-seq and identify three STAT5 binding sites within the Lrrc32 super-enhancer, one shared between $\mathrm{T}_{\text {reg }}$ cells and mammary epithelium and one specific to each respective cell type. Using mice that express only $10 \%$ of normal STAT5 levels we demonstrate the defining contribution of STAT5 in the activation of the Lrrc32 super-enhancer.

\section{Introduction}

Genome-wide approaches, such as whole genome sequencing and ChIP-seq based technologies, have provided researchers with unique handles to understand 
molecular underpinnings of gene regulation and the nature of disease susceptibility loci. It turns out that disease susceptibility loci are frequently found in chromosomal regions that harbor genetic regulatory elements, such as enhancers and superenhancers, and mutations in transcription factor motifs could alter the binding affinities of regulatory proteins ${ }^{5}$. Accurately defining the linear dimensions of enhancers and super-enhancers, the exact positions of transcription factors on the DNA, and the intricacy of the regulatory complex requires high-resolution ChIP-seq technologies.

Nasrallah and colleagues ${ }^{4}$ have investigated the molecular structure and biology of a candidate enhancer in a prominent autoimmune and allergic disease risk locus at chromosome 11q13.5. This enhancer was predicted by the presence of the activating chromatin mark K27-acetylated histone H3 (H3K27ac) in $\mathrm{CD} 4^{+} \mathrm{CD} 127^{-} \mathrm{CD} 25^{+}$regulatory $\mathrm{T}$ cells and other primary lymphocyte lineages. This region is approximately $70 \mathrm{~kb}$ downstream of the LRRC32 gene that encodes GARP, a protein critical for the function of $\mathrm{Foxp}^{+} \mathrm{CD}^{+}$regulatory $\mathrm{T}$ cells, also known as $\mathrm{T}_{\text {reg }}$ cells ${ }^{6}$. Deletion of a $2.3 \mathrm{~kb}$ enhancer fragment from the syntenic mouse region resulted in reduced GARP levels in $T_{\text {reg }}$ cells and the inability to control colitis ${ }^{4}$. A putative binding motif for the cytokine-inducible Signal Transducer and Activator of Transcription (STAT) 5 was identified by DNA sequences and a ChIP-qPCR assay revealed STAT5B binding, suggesting its contribution in the activation of Lrrc32. However, the proposed STAT5 motif (AGGAAATA) is distinctly different from the canonical palindromic GAS motif (TTCNNNGAA). Since ChIP-qPCR monitors transcription factor binding over several hundred nucleotides it is insufficiently precise to accurately pinpoint transcription factor (TF) binding sites. It is therefore possible that STAT5 binds to sequences other than those proposed ${ }^{4}$, or piggybacks on unrelated TFs or even that additional non-STAT TFs might control this enhancer.

\section{Results and Discussion}

To clearly identify and characterize the candidate enhancer linked to the Lrrc32 gene, we turned to high-precision ChIP-seq data from human and mouse and experimental mouse genetics. The transcription factor STAT5A and STAT5B (referred to as STAT5) have non-redundant and essential roles in the physiology of $\mathrm{T}_{\text {reg }}$ cells and mammary epithelial cells ${ }^{7-9}$. Here, by integrating high-quality ChIP-seq, 
RNA-seq and genetic data from $T_{\text {reg }}$ cells ${ }^{10-13}$ and mammary tissue ${ }^{14,15}$, we define the structure of the Lrrc32 enhancer, accurately identify the binding positions of STAT5 and other TFs, and elucidate the significance of STAT5 in regulating Lrrc32 expression.

First, we analyzed ChIP-seq data from human T cells ${ }^{13}$ (Fig. 1). The H3K27ac pattern predicted the presence of several candidate enhancer regions between the 3' ends of the Lrrc32 and Emsy genes. The most prominent one was 70 kb 3' of the Lrrc32 gene (Fig. 1a). This region also featured two sites, E1 and E2, bound by STAT5. Sequences around site E1 are conserved between human and mouse (Fig. 1b) and a STAT5 binding site had been predicted (green highlight in Fig. 1b), next to the SNV that was linked to reduced GARP levels ${ }^{4}$. However, STAT5 does not recognize this site and it rather binds to a GAS (TTCNNNGAA) motif at a distance of 70 bp (yellow highlight in Fig. 1b). STAT5 binding at site E2 also coincides with a GAS motif, the consensus binding motifs for all STAT members except STAT6.

Next, we dug deeper and analyzed the structure and significance of these sites using ChIP-seq data from mouse $\mathrm{T}_{\text {reg }}$ cells ${ }^{10-12}$ and mouse genetics ${ }^{10,15}$. Like in human T cells, STAT5 binding at site E1 coincides with a GAS motif and is located approximately $90 \mathrm{bp}$ apart from the site identified in the Nasrallah study ${ }^{4}$ (Figs. $1 \mathrm{~b}$ and 2). The presence of H3K27ac marks and the H3K4me1 signature, which identifies genuine enhancers, support the regulatory nature of this region. Also, site E2 was present and coincides with all activating histone marks was discovered (Fig. 2a).

Since $T_{\text {reg }}$ cells and mammary epithelium share their absolute dependence on STAT5 $^{7,9}$ we surmised that key enhancers might be shared between these two systems. Using high-quality ChIP-seq data from mouse mammary tissue ${ }^{14,15}$ we identified strong STAT5 binding at two juxtaposed sites (E1 and E3) (Fig. 2a). While site $\mathrm{E} 1$ is shared between $\mathrm{T}_{\text {reg }}$ cells and mammary tissue, site E3 appears to be mammary-specific. Also, site E3 does not coincide with the one identified in the Nasrallah study ${ }^{4}$. However, the TF site identified by Nasrallah et $\mathrm{al}^{4}$ is occupied by two other TF, the glucocorticoid receptor (GR) and nuclear factor I B (NFIB) (on E1 at bottom in Fig. 2a) suggesting these, rather than STAT5, are the occupiers of this enhancer region. The presence of the enhancer-distinctive patterns of H3K27ac and H3K4me1 marks, in particular the histone-depleted sequences surrounding the TF 
binding, and the presence of RNA Polymerase II (Pol II) support the regulatory significance of this region.

Vetting STAT5 and its role in activating the Lrrc32 gene can be accomplished either through the deletion of the GAS motifs or the ablation of the Stat5 genes. Nasrallah ${ }^{4}$ deleted a 2.3 kb fragment spanning enhancer sites E1 and E2 (Fig. 2a), which resulted in an approximately $65 \%$ reduction of $L$ rrc32 expression in $\mathrm{T}_{\text {reg }}$ cells (Fig. 2b). Since this region is extensively covered by activating histone marks and harbors at least two distinct enhancers bound by several TFs, interpretation on the role of STAT5 in Lrrc32 regulation cannot be definitive. We have now addressed the specific contribution of STAT5 in regulating Lrrc32 gene expression in $\mathrm{T}_{\text {reg }}$ cells and mammary tissue through mutant mice that carry different copy numbers of STAT5 genes $^{15}$. Since the combined deletion of Stat5a and Stat5b results in perinatal lethality ${ }^{7,16}$, we generated mice that lack three out of the four Stat5 alleles and carry only a single Stat5a or Stat5b allele (Fig. 2b) ${ }^{15}$. In the presence of only one Stat5a allele, Lrrc32 expression was reduced by $~ 85 \%$ in $\mathrm{T}_{\text {reg }}$ cells ${ }^{10}$ compared to wild type (WT) cells supporting the notion that STAT5 is a key component of the enhancer. Since STAT5B is the more abundant isoform in $\mathrm{T}_{\text {reg }}$ cells, the presence of one copy of Stat5b resulted in a $\sim 55 \%$ reduction of $L$ rrc32 expression. In contrast to $T_{\text {reg }}$ cells, Lrrc32 expression in mammary tissue increased 22-fold in the absence of three out of the four Stat5 alleles (Figs. 2c and d), suggesting that STAT5 might be a contextspecific repressor. Using ChIP-seq from our allele-specific mutants (Fig. 1d) demonstrates the affinity of STAT5 to these sites. In the absence of Stat5a, the two copies of Stat5b are not sufficient to detect Stat5 binding in mammary tissue at sites E1 and E3. Similarly, no Stat5 binding was detected in $\mathrm{T}_{\text {reg }}$ cells in the presence of only two copies of Stat5a. These data support the concept that a specific threshold of STAT5 is needed for the activation of Lrrc32.

Our in-depth analysis revealed a highly complex Lrrc32 super-enhancer with at least three individual STAT5-anchored enhancers, one shared between $T_{\text {reg }}$ and mammary cells, one restricted to mammary epithelium and one specific to $T_{\text {reg }}$ cells. STAT5 binding at a GAS motif in the enhancer shared between $\mathrm{T}_{\text {reg }}$ and mammary cells is structurally distinct from the site proposed by Nasrallah ${ }^{4}$. The binding of GR, NFIB, MED1 and Pol II to the two juxtaposed enhancers in mammary tissue suggests the presence of a multiplex super-enhancer with H3K27ac peaks spanning 
more than $12.5 \mathrm{~kb}$. Similarly, the two enhancers identified in $\mathrm{T}_{\text {reg }}$ cells constitute a super-enhancer.

The establishment and function of $\mathrm{T}_{\text {reg }}$ cells is dependent on both STAT5 ${ }^{9}$ and $\mathrm{GR}^{17}$, and we propose that the enhancer-bound $\mathrm{GR}$ contributes the regulation of Trrc32. Based on mice expressing only one out of the four Stat5 alleles, we propose that STAT5 serves as an anchor to build larger enhancer complexes. Further experiments are needed to identify the significance of the sequence identified by Nasrallah and colleagues ${ }^{4}$ and to establish the function of the other two candidate enhancers identified in our study. It also remains to be determined whether the risk variant rs11236797 abrogates the establishment of a specific enhancer complex. Our work also highlights the need for high-quality and deep-sequenced ChIP-seq data to obtain highly accurate information on the binding position of regulatory proteins to the genome. It remains an enigma why STAT5A/B executes opposite functions in different cell types, repression of Lrrc32 in mammary tissue and activator in $T_{\text {reg }}$ cells.

\section{Methods}

Data availability. All data were obtained from or uploaded to Gene Expression Omnibus (GEO) and Sequence Read Archive (SRA). ChIP-seq data from mammary tissue and $T_{\text {reg }}$ cells were obtained under GSE115370, GSE145193, GSE127144, GSE40930, GSE77656 and DRA005202. RNA-seq data from mammary tissue and $\mathrm{T}_{\text {reg }}$ cells from WT and Stat5 mutant mice were downloaded from GSE37646, GSE77656 and GSE128198.

\section{References}

1. de Lange, K.M. et al. Genome-wide association study implicates immune activation of multiple integrin genes in inflammatory bowel disease. Nat Genet 49, 256-261 (2017).

2. Ferreira, M.A. et al. Shared genetic origin of asthma, hay fever and eczema elucidates allergic disease biology. Nat Genet 49, 1752-1757 (2017).

3. Shevach, E.M. Garp as a therapeutic target for modulation of T regulatory cell function. Expert Opin Ther Targets 21, 191-200 (2017). 
4. Nasrallah, R. et al. A distal enhancer at risk locus 11q13.5 promotes suppression of colitis by Treg cells. Nature (2020).

5. Shi, W., Fornes, O., Mathelier, A. \& Wasserman, W.W. Evaluating the impact of single nucleotide variants on transcription factor binding. Nucleic Acids Res 44, 10106-10116 (2016).

6. Tran, D.Q. et al. GARP (LRRC32) is essential for the surface expression of latent TGF-beta on platelets and activated FOXP3+ regulatory T cells. Proc Natl Acad Sci U S A 106, 13445-50 (2009).

7. Cui, Y. et al. Inactivation of Stat5 in mouse mammary epithelium during pregnancy reveals distinct functions in cell proliferation, survival, and differentiation. Mol Cell Biol 24, 8037-47 (2004).

8. Liu, X. et al. Stat5a is mandatory for adult mammary gland development and lactogenesis. Genes Dev 11, 179-86 (1997).

9. Yao, Z. et al. Nonredundant roles for Stat5a/b in directly regulating Foxp3. Blood 109, 4368-75 (2007).

10. Villarino, A. et al. Signal transducer and activator of transcription 5 (STAT5) paralog dose governs T cell effector and regulatory functions. Elife 5(2016).

11. Villarino, A.V. et al. Subset- and tissue-defined STAT5 thresholds control homeostasis and function of innate lymphoid cells. J Exp Med 214, 2999-3014 (2017).

12. Kitagawa, Y. et al. Guidance of regulatory T cell development by Satb1dependent super-enhancer establishment. Nat Immunol 18, 173-183 (2017).

13. Schmidl, C. et al. The enhancer and promoter landscape of human regulatory and conventional T-cell subpopulations. Blood 123, e68-78 (2014).

14. Lee, H.K., Willi, M., Shin, H.Y., Liu, C. \& Hennighausen, L. Progressing superenhancer landscape during mammary differentiation controls tissue-specific gene regulation. Nucleic Acids Res 46, 10796-10809 (2018).

15. Yamaji, D., Kang, K., Robinson, G.W. \& Hennighausen, L. Sequential activation of genetic programs in mouse mammary epithelium during pregnancy depends on STAT5A/B concentration. Nucleic Acids Res 41, 1622 36 (2013).

16. Yao, Z. et al. Stat5a/b are essential for normal lymphoid development and differentiation. Proc Natl Acad Sci U S A 103, 1000-5 (2006). 
17. Rocamora-Reverte, L. et al. Glucocorticoid Receptor-Deficient Foxp3(+) Regulatory T Cells Fail to Control Experimental Inflammatory Bowel Disease. Front Immunol 10, 472 (2019).

\section{Acknowledgements}

This work was supported by the Intramural Research Programs (IRPs) of National Institute of Diabetes and Digestive and Kidney Diseases (NIDDK) and utilized the computational resources of the NIH HPC Biowulf cluster (http://hpc.nih.gov).

\section{Author contributions}

H.K.L. and L.H. designed the study. H.K.L. performed computational analysis. All authors wrote the manuscript and approved the final version.

\section{Competing interests}

The authors declare no competing financial interests. 
a
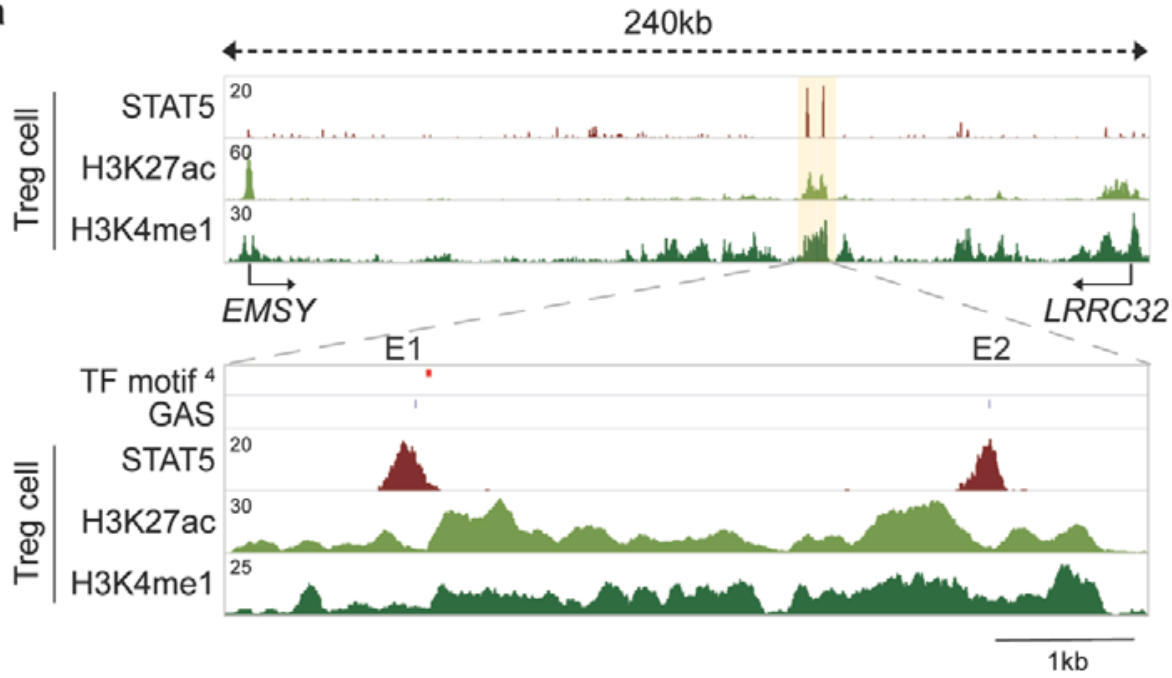

b

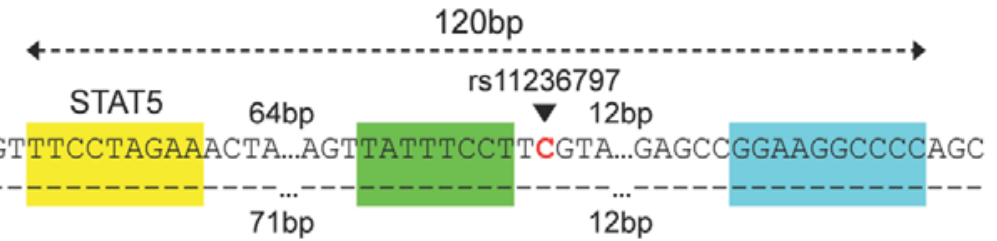

Fig. 1 Conserved transcription factor binding motifs in the LRRC32 enhancer region. a, ChIP-seq profiles showed STAT5 binding at the LRRC32 locus and the candidate enhancers in human $T_{\text {reg }}$ cells. The orange shades indicate candidate regulatory regions. The canonical GAS motifs are indicated by a blue marks (E1 and E2). The 'STAT site' identified by Nasrallah ${ }^{4}$ is indicated by a red mark. E1 and E2 are putative enhancers with strong STAT5 binding in human $T_{\text {reg }}$ cells. b, Genomic sequence alignment (hg19 and mm10) shows the conserved canonical STAT5 binding motif (GAS) in yellow, the 'STAT5 site' identified by Nasrallah ${ }^{4}$ highlighted in green and the NF-KB binding motif (Nasrallah ${ }^{4}$ ) highlighted in turquois. 
bioRxiv preprint doi: https://doi.org/10.1101/2020.06.13.150177; this version posted June 15, 2020. The copyright holder for this preprint (which was not certified by peer review) is the author/funder. This article is a US Government work. It is not subject to copyright under 17 USC 105 and is also made available for use under a CCO license.

a
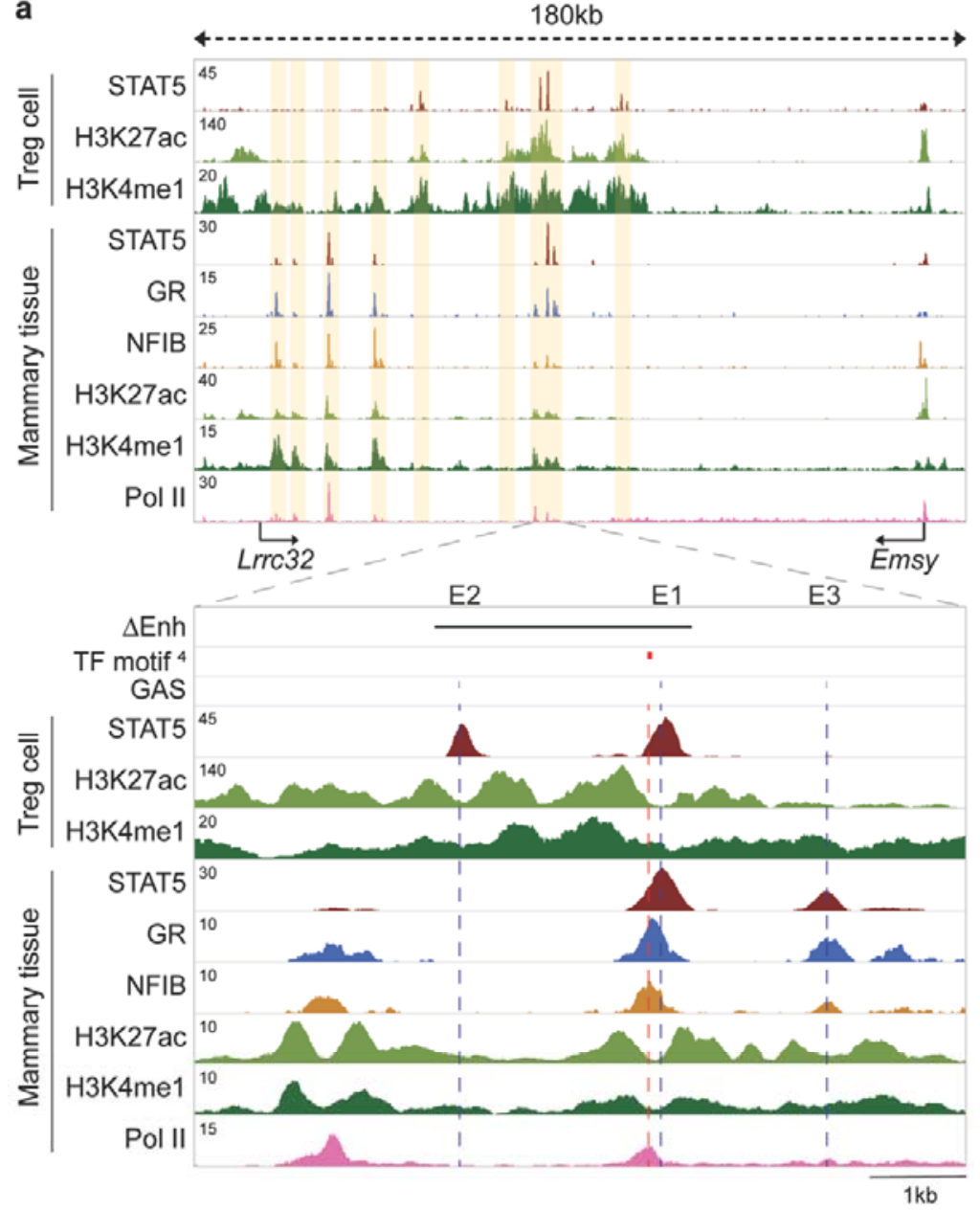

b

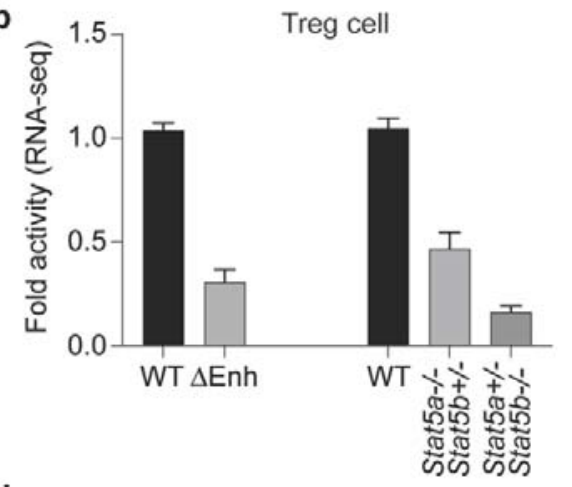

C

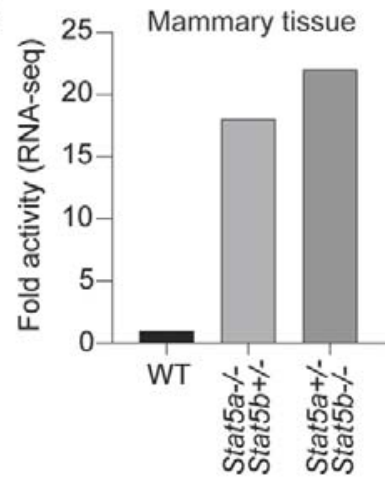

d E2 E1 E3

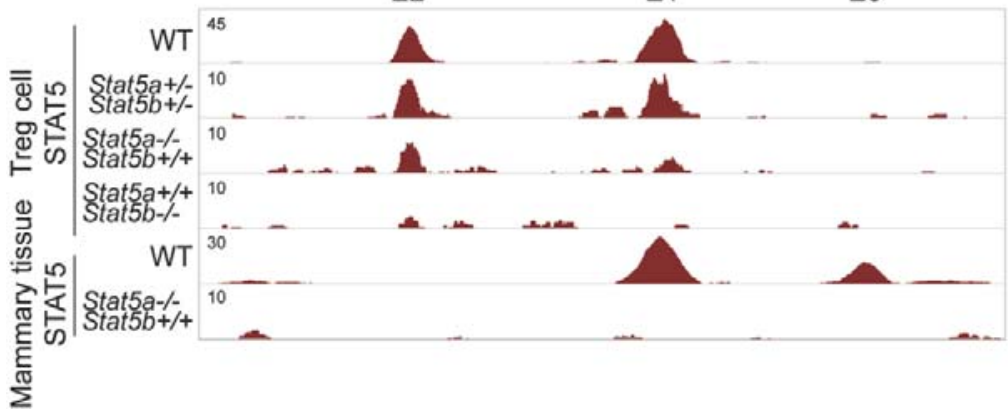


Fig. 2 Establishment of putative Lrrc32 enhancers in mammary epithelium and regulatory $\mathbf{T}$ ( $\left.\mathbf{T}_{\text {reg }}\right)$ cells. a, ChIP-seq data for STAT5A (in mammary tissue) or panSTAT5 (in IL2-stimulated CD4+ T cells), GR, NFIB, Pol II and histone marks H3K27ac and H3K4me1, provide structural information of the locus between the Lrrc32 and Emsy genes in lactating mammary tissue and activated $\mathrm{T}_{\text {reg }}$ cells. Solid arrows indicate the orientation of genes. The orange shades highlight candidate regulatory regions. The black bar indicates the deleted enhancer region $(\Delta \mathrm{Enh})^{4}$. The blue broken lines (E1, E2 and E3) highlight STAT5 binding associated with the canonical GAS motif. The red line depicts the 'STAT site' identified by Nasrallah". E1, E2 and E3 are putative enhancers with strong STAT5 binding. b, RNA-seq data from Nasrallah ${ }^{4}$ and Villarino ${ }^{10}$ demonstrate the relative expression of Lrrc32 gene in $T_{\text {reg }}$ cells in the absence of the $2.3 \mathrm{~kb}$ enhancer region and different Stat5 alleles, respectively. c, Lrrc32 mRNA levels in mouse mammary tissue of WT and Stat5 mutant mice were measured by RNA-seq ${ }^{15}$. $\mathbf{d}$, The putative enhancers were profiled using ChIP-seq data of WT and mutant tissue. 